\title{
Exponentiated Rayleigh Distribution: A Bayes Study Using MCMC Approach Based on Unified Hybrid Censored Data
}

\author{
M. G. M. Ghazal ${ }^{1}$, H. M. Hasaballah² \\ ${ }^{1}$ Mathematics Department, Faculty of Science, Minia University \\ El-Minia, Egypt. \\ eghazal39@yahoo.com \\ ${ }^{2}$ Mathematics Department, Faculty of Science, Minia University \\ El-Minia, Egypt. \\ hasaballahmohamed@yahoo.com
}

\begin{abstract}
This paper aims to estimate the unknown parameters, survival and hazard functions for exponentiated Rayleigh distribution based on unified hybrid censored data. The maximum likelihood estimators (MLEs), Bayes, and parametric bootstrap methods are used for estimating the two unknown parameters as well as survival and hazard functions. We propose to apply Markov chain Monte Carlo (MCMC) technique to carry out a Bayesian estimation procedure. Approximate confidence intervals for the unknown parameters moreover survival and hazard functions are constructed based on the s-normal approximation to the asymptotic distribution of MLEs. The approximate Bayes estimators have been obtained under the assumptions of informative and non-informative priors depending on symmetric and asymmetric loss functions via the Gibbs within Metropolis-Hasting samplers procedure. Finally, the proposed methods can be understood through illustrating the results of the real data analysis.
\end{abstract}

\section{KEYWORDS}

Exponentiated Rayleigh distribution; Unified hybrid censoring scheme; Maximum likelihood estimators; Bootstrap method; Bayesian estimation; MCMC method.

\section{INTRODUCTION}

Supposing there are $n$ identical units put to test in the same time, when the experiment is terminated at apre-fixed time $T$, we refer to Type-I censoring scheme. The disadvantage of Type-I censoring scheme is very few failures may occur before time $T$. The experiment is terminated when the $r^{\text {th }}$ failures occur, we refer to Type-Il censoring scheme. Furthermore, the disadvantage of Type-Il censoring scheme is that the termination time is unknown prior to the experiment. For more details on Type-I and Type-II censoring, and related inferential issues, see Nelson [23] and Balakrishnan and Cohen [4]. A hybrid censoring scheme (HCS) is a mixture of Type-I and Type-II censoring schemes. This HCS was introduced by Epstein [10], to avoid the disadvantages of Type-I and type-II censored scheme, and it has been used in reliability acceptance test in MILSTD-781-C [21], see also Jeong et al. [16], Gupta and Kundu [12], Park and Balakrishnan [24]. Epstein [10] is considered a hybrid censored scheme in which the life-testing experiment is terminated at a random time $T^{\star}=\min \left\{X_{r: n}, T\right\}$, this refer to Type-I HCS. The disadvantage of Type-I HCS is very few failures occurring until the pre-fixed time T. To overcome this disadvantage, Childs et al. [8] introduced a substitute Type-I HCS that would terminate the experiment at the random time $T^{*}=\max \left\{X_{r: n}, T\right\}$. This HCS is called Type-II HCS. In the same respect, to avoid the disadvantages in these schemes, Chandrasekar et al. [7] proposed two new schemes which are called generalized Type-I and Type-II HCS. In generalized Type-I HCS, fix $k, r \in(1,2, \ldots, \mathrm{n})$ and $\mathrm{T} \in(0, \infty)$ such that $k<r<n$. If the $k^{\text {th }}$ failure occurs before time $T$, the experiment is terminated at $\min \left\{X_{r: n} ; T\right\}$. If the $k^{\text {th }}$ failure occurs after time $T$, the experiment is terminated at $X_{k: n}$, so, it is clear that this HCS modifies the Type-I HCS by allowing the experiment to continue after time $T$ if very few failures had observed until that time point. In generalized Type-II HCS, fix $r \in(1,2, \ldots, n)$ and $T_{1}, T_{2} \in(0, \infty)$ such that $T_{2}>T_{1}$. If the $r^{\text {th }}$ failure occurs before time point $T_{1}$, the experiment is terminated at $T_{1}$. If the $r^{\text {th }}$ failure occurs between $T_{1}$ and $T_{2}$, the experiment is terminated at $X_{r: n}$. If the $r^{\text {th }}$ failure occurs after $T_{2}$, the experiment is terminated at $T_{2}$. There are some drawbacks in generalized HCS, such as, in generalized Type-I HCS. Moreover, because the experiment is terminated at the same time or before $T$, we cannot guarantee observing $r$ failures. While in the generalized Type-II HCS, we cannot observe any failure at all or observe only few number of failures until the pre-fixed time $T_{2}$. To avoid these drawbacks, Balakrishnan et al. [5] introduced an unified hybrid censoring scheme (UHCS), which can be described as follows, fix $r, k \in\{1, \ldots, n\}$ where $k<r<n$ and $T_{1}, T_{2} \in(0, \infty)$ where $T_{2}>T_{1}$. If the $k^{\text {th }}$ failure occurs before time $T_{1}$, the experiment is terminated at $\min \left\{\max \left\{X_{r: n}, T_{1}\right\}, T_{2}\right\}$. If the $k^{\text {th }}$ failure occurs between $T_{1}$ and $T_{2}$, the experiment is terminated at $\min \left\{X_{r: n}, T_{2}\right\}$. and if the $k^{\text {th }}$ failure occurs after time $T_{2}$, the experiment is terminated at $X_{k: n}$. Under this censoring scheme, we can guarantee that the experiment would be completed at most in time $T_{2}$ with at least $k$ failure and if not, we can guarantee exactly $k$ failures.

Therefore, under the UHCS, we have one of the following six cases:

Case I: $0<x_{k: n}<x_{r: n}<T_{1}<T_{2}$; the experiment is terminated at $T_{1}$.

Case II: $0<x_{k: n}<T_{1}<x_{r: n}<T_{2}$, the experiment is terminated at $x_{r: n}$.

Case III: $0<x_{k: n}<T_{1}<T_{2}<x_{r: n}$, the experiment is terminated at $T_{2}$.

Case IV: $0<T_{1}<x_{k: n}<x_{r: n}<T_{2}$, the experiment is terminated at $x_{r: n}$.

Case $\mathrm{V}: 0<T_{1}<x_{k: n}<T_{2}<x_{r: n}$, the experiment is terminated at $T_{2}$. 
Case VI: $0<T_{1}<T_{2}<x_{k: n}<x_{r: n}$, the experiment is terminated at $x_{k: n}$.

The Rayleigh distribution is an important distribution in statistics and operations research. It has a wide range of applications in several areas such as health, agriculture, biology, analyzing wind speed data and other. Burr [6] introduced twelve different forms of cumulative distribution functions for modeling lifetime data. The exponentiated Rayleigh distribution was derived by Vodã [35] to generalize the Rayleigh distribution originally developed by Rayleigh [28]. One of the important properties of the Rayleigh distribution is that its failure rate is an increasing function of time. Exponentiated Rayleigh distribution was studied by Sartawi and Abu-Salih [29], Jaheen [15, 14], Ahmad et al. [2], Raqab [25] and Surles and Padgett [31]. Recently, Surles and Padgett [32], introduced two parameter Burr Type $X$ distribution and correctly named as the exponentiated Rayleigh distribution. The two parameter exponentiated Rayleigh distribution is a special case of the Weibull distribution originally suggested by Mudholkar and Srivastava [22]. Kundu and Raqab [17] estimated the parameters of $\operatorname{ER}(\alpha, \beta)$ distribution by using different methods. Raqab and Madi [26] studied exponentiated Rayleigh distribution in Bayesian framework. Abd-Elfattah [1] discussed the goodness of fit test for the generalized Rayleigh distribution with unknown parameters. Raqab and Madi [27] discussed the inference for the generalized Rayleigh distribution based on progressively censored data. Lio et al. [18] discussed Parameter estimations for generalized Rayleigh distribution under progressively Type-I interval censored data. Fathipour et al. [11] studied the estimating $R=$ $P(Y<X)$ in the generalized Rayleigh distribution with different scale parameters. Al Kanani and Jasim [3] discussed nonBayesian and Bayesian estimation for generalized Rayleigh distribution. Shrestha and Kumar[30] studied Bayesian analysis for the exponentiated Rayleigh distribution. Mahmoud and Ghazal [19] studied estimations from the exponentiated Rayleigh distribution based on generalized Type-II hybrid censored data.

The cumulative distribution function (CDF) given by

$F(x ; \alpha, \beta)=\left(1-e^{-\beta x^{2}}\right)^{\alpha}, \quad x>0, \alpha, \beta>0$.

Where $\alpha$ and $\beta$ are the shape and scale parameters respectively, the probability density function (PDF) is

$f(x ; \alpha, \beta)=2 \alpha \beta x e^{-\beta x^{2}}\left(1-e^{-\beta x^{2}}\right)^{\alpha-1}, \quad x>0, \alpha, \beta>0$.

The Survival function $S(t)$ is given by

$$
S(t)=1-\left(1-e^{-\beta t^{2}}\right)^{\alpha}, \quad t>0 .
$$

The hazard function $H(t)$ is given by

$$
H(t)=\frac{2 \alpha \beta t e^{-\beta t^{2}}\left(1-e^{-\beta t^{2}}\right)^{\alpha-1}}{1-\left(1-e^{-\beta t^{2}}\right)^{\alpha}}, \quad t>0 .
$$

The rest of the paper is organized as follows: In Section 2, we discussed the MLEs of the unknown parameters in addition to $S(t)$ and $H(t)$. In Section 3, credible intervals based the MLEs are presented. In Section 4, confidence intervals were obtained from two parametric bootstrap procedures. In Section 5, we apply MCMC technique to obtain the confidence intervals. One data set has been analyzed for illustrative purposes in Section 6. Finally, conclusions are given in Section 7.

\section{MAXIMUM LIKELIHOOD ESTIMATION}

In this section, we obtained the $\operatorname{MLEs}$ of $\operatorname{ER}(\alpha, \beta)$ distribution when $\alpha$ and $\beta$ are unknown. Let $\left(x_{1}, \ldots, x_{n}\right)$ be a random sample of size $\mathrm{n}$ from $\operatorname{ER}(\alpha, \beta)$ distribution, then the likelihood function for six cases of the UHCS is as follows:

$$
\begin{aligned}
& L(\underline{x}, \theta)=\frac{n !}{(n-R) !}\left[\prod_{i=1}^{R} f\left(x_{i}\right)\right][1-F(C)]^{n-R} . \\
& R, C)= \begin{cases}\left(d, T_{1}\right), & \text { for case-I, } \\
\left(r, x_{r: n}\right) & \text { for case-II and case-IV, } \\
\left(d_{2}, T_{2}\right), & \text { for case-III and for case-V, } \\
\left(k, x_{k: n}\right), & \text { for case-VI. }\end{cases}
\end{aligned}
$$

Where $R$ indicates the number of the total failures in experiment up to time $C$ (the stopping time point) and $d_{1}$ and $d_{2}$ indicate the number of failures that occur before time points $T_{1}$ and $T_{2}$, respectively. From (1.1), (1.2) and (2.1) we get 
$L(\underline{x} ; \alpha, \beta)=K \alpha^{R} \beta^{R} \prod_{i=1}^{R} x_{i} e^{-\beta \sum_{i=1}^{R} x_{i}^{2}} \prod_{i=1}^{R}\left[1-e^{-\beta x_{i}^{2}}\right]^{\alpha-1}\left[1-\left(1-e^{-\beta C^{2}}\right)^{\alpha}\right]^{n-R}$.

Where $K=\frac{2^{R} n !}{(n-R) !}$.

The logarithm of the likelihood function can be written as

$$
\begin{aligned}
\ell(\underline{x} ; \alpha, \beta)= & \ln (K)+R \ln (\alpha)+R \ln (\beta)+\sum_{i=1}^{R} \ln \left(x_{i}\right)-\beta \sum_{i=1}^{R} x_{i}^{2} \\
& +(\alpha-1) \sum_{i=1}^{R} \ln \left(1-e^{-\beta x_{i}^{2}}\right)+(n-R) \ln \left[1-\left(1-e^{-\beta C^{2}}\right)^{\alpha}\right] .
\end{aligned}
$$

Applying the first derivative with respect to $\alpha$ and $\beta$ in (2.3) and equating by zero, then we get the two normal equations as follows

$$
\begin{aligned}
& \frac{\partial \ell}{\partial \alpha}=\frac{R}{\alpha}+\sum_{i=1}^{R} \ln \left(1-e^{-\beta x_{i}^{2}}\right)-\frac{(n-R)\left(1-e^{-\beta C^{2}}\right)^{\alpha} \ln \left(1-e^{-\beta C^{2}}\right)}{1-\left(1-e^{-\beta C^{2}}\right)^{\alpha}}=0, \\
& \frac{\partial \ell}{\partial \beta}=\frac{R}{\beta}-\sum_{i=1}^{R} x_{i}^{2}+(\alpha-1) \sum_{i=1}^{R} \frac{x_{i}^{2} e^{-\beta x_{i}^{2}}}{1-e^{-\beta x_{i}^{2}}}-\frac{\alpha(n-R)\left(1-e^{-\beta C^{2}}\right)^{\alpha-1} C^{2} e^{-\beta C^{2}}}{1-\left(1-e^{-\beta C^{2}}\right)^{\alpha}}=0 .
\end{aligned}
$$

$R$ and $C$ are defined above. The MLEs of $\alpha$ and $\beta$ can be found by solving the system of equations (2.4) and (2.5), even if the suggested estimators cannot be expressed in closed forms, we can use a suitable numerical technique to obtain the estimators. Moreover, we can obtain the MLEs of $S(t)$ and $H(t)$ after replacing $\alpha$ and $\beta$ by their MLEs $\hat{\alpha}$ and $\hat{\beta}$ as following

$$
\begin{aligned}
\hat{S}_{M L}(t) & =1-\left(1-e^{-\hat{\beta} t^{2}}\right)^{\hat{\alpha}}, \\
\hat{H}_{M L}(t) & =\frac{2 \hat{\alpha} \hat{\beta} t e^{-\beta} t^{2}\left(1-e^{-\beta t^{2}}\right)^{\hat{\alpha}-1}}{1-\left(1-e^{-\hat{\beta} t^{2}}\right)^{\hat{\alpha}}} .
\end{aligned}
$$

\section{CONFIDENCE INTERVAL}

The asymptotic variance-covariance of the MLEs for parameters $\alpha$ and $\beta$ are given by elements of the inverse of the Fisher information matrix are defined as

$$
I_{i j}=-E\left(\frac{\partial^{2} \ell}{\partial \theta_{i} \partial \theta_{j}}\right), \quad \text { where } i, j=1,2, \quad \theta_{1}=\alpha \text { and } \quad \theta_{2}=\beta .
$$

Unluckily, we found difficult to solve the above expectations. Therefore, we gave the approximate asymptotic variancecovariance matrix for the MLEs, which, can be obtained by

$$
I^{-1}(\alpha, \beta)=\left(\begin{array}{cc}
-\frac{\partial^{2} \ell}{\partial \alpha^{2}} & -\frac{\partial^{2} \ell}{\partial \alpha \partial \beta} \\
-\frac{\partial^{2} \ell}{\partial \beta \partial \alpha} & -\frac{\partial^{2} \ell}{\partial \beta^{2}}
\end{array}\right)_{(\hat{\alpha}, \hat{\beta})}^{-1}=\left(\begin{array}{cc}
\widehat{\operatorname{var}}(\hat{\alpha}) & \operatorname{cov}(\hat{\alpha}, \hat{\beta}) \\
\operatorname{cov}(\hat{\beta}, \hat{\alpha}) & \widehat{\operatorname{var}}(\hat{\beta})
\end{array}\right),
$$


where

$$
\begin{gathered}
\frac{\partial^{2} \ell}{\partial \alpha^{2}}=-\frac{R}{\alpha^{2}}-\frac{(n-R) \ln \left(1-e^{-\beta C^{2}}\right)^{2}\left(1-e^{-\beta C^{2}}\right)^{\alpha}}{\left[1-\left(1-e^{-\beta C^{2}}\right)^{\alpha}\right]^{2}}, \\
\frac{\partial^{2} \ell}{\partial \beta^{2}}=\frac{-R}{\beta^{2}}+(\alpha-1) \sum_{i=1}^{R} \frac{-\left(1-e^{-\beta x_{i}^{2}}\right) x_{i}^{4} e^{-\beta x_{i}^{2}}-x_{i}^{4} e^{-2 \beta x_{i}^{2}}}{\left(1-e^{-\beta x_{i}^{2}}\right)^{2}} \\
-\alpha(n-R) C^{2}\left[\frac{\left(1-\left(1-e^{-\beta C^{2}}\right)^{\alpha}\right)\left[(\alpha-1)\left(1-e^{-\beta C^{2}}\right)^{\alpha-2} C^{2} e^{-2 C^{2} \beta}-C^{2} e^{-\beta C^{2}}\left(1-e^{-\beta C^{2}}\right)^{\alpha-1}\right]}{\left[1-\left(1-e^{-\beta C^{2}}\right)^{\alpha}\right]^{2}}\right. \\
\left.+\frac{\alpha C^{4} e^{-2 \beta C^{2}}\left(1-e^{-\beta C^{2}}\right)^{2 \alpha-2}}{\left[1-\left(1-e^{-\beta C^{2}}\right)^{\alpha}\right]^{2}}\right], \\
\frac{\partial^{2} \ell}{\partial \beta \partial \alpha}=\frac{\partial^{2} \ell}{\partial \alpha \partial \beta}=\sum_{i=1}^{R} \frac{x_{i}^{2} e^{-\beta x_{i}^{2}}}{1-e^{-\beta x_{i}^{2}}}-C^{2}(n-R) e^{-\beta C^{2}}\left[1-e^{-\beta C^{2}}\right]^{\alpha-1}[ \\
\left.\times \frac{\left(1-\left(1-e^{-\beta C^{2}}\right)^{\alpha}\right)\left[1+\alpha \ln \left(1-e^{-\beta C^{2}}\right)\right]+\alpha\left(1-e^{-\beta C^{2}}\right)^{\alpha} \ln \left(1-e^{-\beta C^{2}}\right)}{\left[1-\left(1-e^{-\beta C^{2}}\right)^{\alpha}\right]^{2}}\right] .
\end{gathered}
$$

The asymptotic normality of the MLEs can be used to compute the approximate confidence intervals (ACI) for parameters $\alpha$ and $\beta$. Therefore, $(1-\gamma) 100 \%$ confidence intervals for parameters $\alpha$ and $\beta$ become

$$
\left(\hat{\alpha} \pm Z_{\gamma / 2} \sqrt{\widehat{\operatorname{var}}(\hat{\alpha})}\right) \quad \text { and } \quad\left(\hat{\beta} \pm Z_{\gamma / 2} \sqrt{\widehat{\operatorname{var}}(\hat{\beta})}\right) \text {. }
$$

Where $Z_{\gamma / 2}$ is a standard normal variate.

Moreover; to find the $\mathrm{ACl}$ of the $S(t)$ and $H(t)$, we need to find the variances of them. To find the approximate estimates of the variance of $\hat{S}(t)$ and $\hat{H}(t)$ we use the delta method. The delta method is a general approach for computing confidence intervals for functions of MLEs. Depending on this method, the variance of $\hat{S}(\mathrm{t})$ and $\hat{H}(t)$ respectively given by

$$
\hat{\sigma}_{S(t)}^{2}=[\nabla \hat{S}(t)]^{T}[\hat{V}][\nabla \hat{S}(t)] \quad \text { and } \quad \hat{\sigma}_{H(t)}^{2}=[\nabla \hat{H}(t)]^{T}[\hat{V}][\nabla \hat{H}(t)] \text {, where } \nabla \hat{S}(t) \text { and }
$$

$\nabla \hat{\mathrm{H}}(\mathrm{t})$ are the gradient of $\hat{\mathrm{S}}(\mathrm{t})$ and $\hat{\mathrm{H}}(\mathrm{t})$ respectively, with respect to $\alpha$ and $\beta$. And $\hat{V}=I^{-1}(\alpha, \beta)$. Therefore, $(1-\gamma) 100 \%$ confidence intervals for $S(t)$ and $H(t)$ become

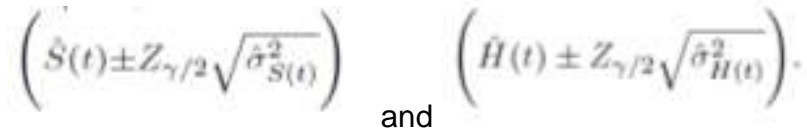

\section{BOOTSTRAP CONFIDENCE INTERVALS}

In this section, two parametric bootstrap procedures are provided to construct the bootstrap confidence intervals of $\alpha, \beta$, $S(t)$ and $H(t)$. The first one is the percentile bootstrap (Boot-p) confidence interval proposed by Efron[9]. The second one is the bootstrap-t (Boot-t) confidence interval proposed by Hall [13]. The algorithms for estimating the confidence intervals using both methods are illustrated as follows.

\subsection{The procedure of Boot-p method}


1. From the original sample $\underline{x}=\left(x_{1 ; n}, x_{2 ; n}, \ldots, x_{R ; n}\right)$ compute the ML estimates of the parameters $\alpha$ and $\beta$ from equations (2.4) and (2.5).

2. Using the values of $\hat{\alpha}$ and $\hat{\beta}$ in equations (1.3) and (1.4), we obtain the ML estimates $\hat{S}(t)$ and $\hat{H}(t)$.

3. Get a bootstrap sample $\underline{x^{*}}=\left(x_{1 ; n}^{*}, x_{2 ; n}^{*}, \ldots, x_{R ; n}^{*}\right)$ by resampling with replacement.

4. As in step 1, based on $x^{*}$ compute the bootstrap sample estimates of $\eta$ where $\eta=[\alpha, \beta, S(t), H(t)]$, say $\hat{n}^{*}=\left\lfloor\hat{\alpha}^{*}, \hat{\beta}^{*}, \hat{S} *(t), \hat{H} *(t)\right\rfloor$

5. Repeat steps 3 and $4 \mathrm{~N}$ Boot times, and obtain $\hat{\eta}_{1}^{*}, \hat{\eta}_{2}^{*}, \ldots ., \hat{\eta}_{N B o o t}^{*}$, where $\hat{\eta}_{i}^{*}=\left\lfloor\hat{\alpha}_{i}^{*}, \hat{\beta}_{i}^{*}, \hat{S}_{i}^{*}(t), \hat{H}_{i}^{*}(t)\right\rfloor i=1,2, \ldots . . . N B o o t$.

6. Arrange $\hat{\eta}_{i}^{*}, i=1,2, \ldots, N B o o t$ in an ascending order to obtain the bootstrap sample $\left(\hat{\eta}_{(1)}^{*}, \hat{\eta}_{(2)}^{*}, \ldots . ., \hat{\eta}_{(N B o o t)}^{*}\right)$.

7. Let $G_{1}(x)=p\left(\eta_{i} \leq x\right)$ be the CDF of $\eta_{i}$. Define $\eta_{i B o o t}=G_{1}^{-1}(x)$ for given $0<x<1$. The approximate bootstrap $100(1-\gamma) \%$ confidence interval of $\eta_{i}$ is given by $\left[\eta_{i B o o t-p}\left(\frac{\gamma}{2}\right), \eta_{i B o o t-p}\left(1-\frac{\gamma}{2}\right)\right]$.

\subsection{The procedure of Boot-t method}

1. From the original sample $\underline{x}=\left(x_{1 ; n}, x_{2 ; n}, \ldots, x_{R ; n}\right)$ compute the $\mathrm{ML}$ estimates of the parameters: $\alpha$ and $\beta$ from equations (2.4) and (2.5).

2. Using the values of $\hat{\alpha}$ and $\hat{\beta}$ in equation (1.3) and (1.4), we obtain the ML estimates $\hat{S}(t)$ and $\hat{H}(t)$.

3. Get a bootstrap sample $\underline{x^{*}}=\left(x_{1 ; n}^{*}, x_{2 ; n}^{*}, \ldots, x_{R ; n}^{*}\right)$ by resampling with replacement. $\hat{\alpha}$ and $\hat{\beta}$ in step 1 to generate a bootstrap sample.

4. As in step 1, based on $\underline{x}$ compute the bootstrap sample estimates of $\eta$ where $\eta=[\alpha, \beta, S(t), H(t)]$ say $\hat{n}^{*}=\left\lfloor\hat{\alpha}^{*}, \hat{\beta}^{*}, \hat{S} *(t), \hat{H} *(t)\right\rfloor$

5. Compute the $T^{*} \eta$ statistic defined as

$$
T^{* \eta}=\frac{\hat{\eta}^{*}-\hat{\eta}}{\sqrt{\hat{\operatorname{var}}\left(\hat{\eta}^{*}\right)}}
$$

Where $\operatorname{var}\left(\hat{\eta}^{*}\right)$ are obtained by the Fisher information matrix.

6. Repeat step 3 and 5 NBoot times and obtain $T_{1}^{* \eta}, T_{2}^{* \eta}, \ldots ., T_{N B o o t}^{*} \eta$

7. Arrange $T_{1}^{* \eta}, T_{2}^{* \eta}, \ldots, T_{N B o o t}^{*}$, , in an ascending orders and obtain the ordered sequences $\left(\hat{T}_{(1)}^{* \eta}, \hat{T}_{(2)}^{* \eta}, \ldots . ., \hat{T}_{(N B o o t}^{* \eta}\right)$.

8. Let $G_{2}(x)=p\left(T^{*} \leq x\right)$ be the CDF $T^{*}$. For a given $x$, define $\hat{\eta}_{\text {boot }-t}(x)=\hat{\eta}+G_{2}^{-1}(x) \sqrt{\hat{\operatorname{var}}(\hat{\eta})} \cdot$ Then the $100(1-\gamma) \%$ percentile confidence interval of $\eta=[\alpha, \beta, S(t), H(t)]$ is $\left[\hat{\eta}_{i \text { Boot }-p}\left(\frac{\gamma}{2}\right), \hat{\eta}_{i \text { Boot }-p}\left(1-\frac{\gamma}{2}\right)\right]$.

\section{BAYES ESTIMATION}

In this section, we described how to obtain the Bayes estimators and the corresponding credible intervals of parameters $\alpha$, $\beta, S(t)$ and $H(t)$ of $E R(\alpha, \beta)$ distribution under squared error loss, LINEX loss and general entropy loss functions, based on UHCS when $\alpha$ and $\beta$ are supposed to be unknown. We assume that $\alpha$ and $\beta$ have the following gamma prior distributions 


$$
\begin{array}{ll}
\pi_{1}(\alpha) \propto \alpha^{a_{1}-1} e^{-b_{1} \alpha}, & \alpha>0, \\
\pi_{2}(\beta) \propto \beta^{a_{2}-1} e^{-b_{2} \beta}, & \beta>0 .
\end{array}
$$

Here all the hyper parameters $a_{1} ; a_{2} ; b_{1}$ and $b_{2}$ are assumed to be known and non-negative.

The joint prior distribution for $\alpha$ and $\beta$ is

$$
\pi(\alpha, \beta) \propto \alpha^{a_{1}-1} \beta^{a_{2}-1} e^{-\left(b_{1} \alpha+b_{2} \beta\right)} .
$$

Using the joint prior distribution of $\alpha$ and $\beta$, we obtained the joint distribution of the data, $\alpha$ and $\beta$ as

$$
L(\underline{x} \mid \alpha, \beta) \times \pi_{1}(\alpha) \times \pi_{2}(\beta) .
$$

Based on (5.15), the joint posterior density function of $\alpha$ and $\beta$ can be written as

$$
\pi^{*}(\alpha, \beta \mid \underline{x})=\frac{L(\underline{x} \mid \alpha, \beta) \times \pi_{1}(\alpha) \times \pi_{2}(\beta)}{\int_{0}^{\infty} \int_{0}^{\infty} L(\underline{x} \mid \alpha, \beta) \times \pi_{1}(\alpha) \times \pi_{2}(\beta) d \alpha d \beta} .
$$

Therefore, the Bayes estimator of a function $u(\alpha, \beta)$ under the squared error loss function is as follows

$$
u_{\hat{B} S}(\alpha, \beta)=E[u(\alpha, \beta) \mid \underline{x}]=\int_{\alpha} \int_{\beta} u(\alpha, \beta) \pi^{*}(\alpha, \beta \mid \underline{x}) d \alpha d \beta .
$$

The Bayes estimator of a function $u(\alpha, \beta)$ under the LINEX loss function is as follows

$$
u_{B}(\alpha, \beta)=-\frac{1}{a} \ln \left(E\left[e^{-a u(\alpha, \beta)} \mid \underline{x}\right]\right)=-\frac{1}{a} \ln \left(\int_{\alpha} \int_{\beta} e^{-a u(\alpha, \beta)} \pi^{*}(\alpha, \beta \mid \underline{x}) d \alpha d \beta\right) .
$$

The Bayes estimator of a function $u(\alpha, \beta)$ under the General entropy loss function is as follows

$$
u_{\hat{B} G}(\alpha, \beta)=\left[E\left[u(\alpha, \beta)^{-a} \mid \underline{x}\right]\right]^{-\frac{1}{a}}=\left(\int_{\alpha} \int_{\beta}[u(\alpha, \beta)]^{-a} \pi^{*}(\alpha, \beta \mid \underline{x}) d \alpha d \beta\right)^{-\frac{1}{a}} .
$$

It is very difficult to compute the equations (5.17), (5.18) and (5.19) analytically. Then, we suggested using MCMC to generate samples from (5.20) and approximate these equations under square error loss, LINEX loss and general entropy loss functions. The MCMC method is used for computing the Bayes estimates of the parameter $\alpha$ and $\beta$ in addition to $S(t)$ and $H(t)$ and also to construct the corresponding credible intervals based on the generated posterior sample. For more details about the MCMC methods see, for example, Upadhyay et al.[33] and Upadhyay and Gupta [34]. We supposed the Gibbs with in Metropolis sampler, which requires the derivation of the complete. From (5.16), the joint posterior up to proportionality can be written as

$$
\begin{gathered}
\pi^{*}(\alpha, \beta \mid \underline{x}) \propto \alpha^{a_{1}+R-1} \beta^{a_{2}+R-1} e^{-\beta\left(b_{2}+\sum_{i=1}^{R} x_{i}^{2}\right)} e^{-\alpha\left[b_{1}-\sum_{i=1}^{R} \ln \left(1-e^{-\beta x_{i}^{2}}\right)\right]} \\
e^{-\sum_{i=1}^{R} \ln \left(1-e^{-\beta x_{2}^{2}}\right)}\left[1-\left(1-e^{-\beta C^{2}}\right)^{\alpha}\right]^{n-R}
\end{gathered}
$$

From (5.20) we get the posterior density function of $\alpha$ given $\beta$

$$
\pi_{1}^{*}(\alpha \mid \beta, \underline{x}) \propto \alpha^{a_{1}+R-1} e^{-\alpha\left[b_{1}-\sum_{i=1}^{R} \ln \left(1-e^{-\beta x_{i}^{2}}\right)\right]} .
$$


Also the posterior density function of $\beta$ given $\alpha$ can be written as

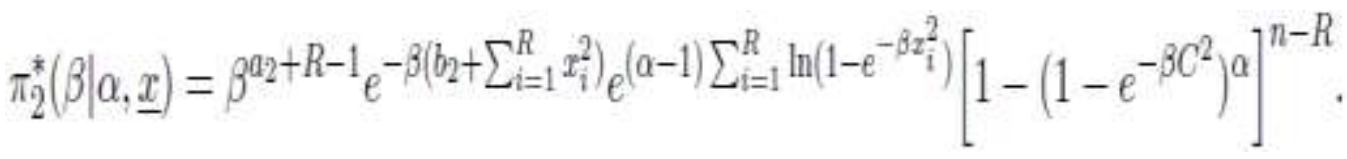

Therefore, the posterior density function of $\alpha$ given $\beta$ is gamma with the shape parameter $\left(\alpha_{1}+R\right)$ and scale parameter $\left(b_{1}-\sum_{i=1}^{R} \ln \left(1-e^{-\beta x_{i}^{2}}\right)\right)$ and, therefore, samples of $\alpha$ can be easily generated using any gamma generating routine. Furthermore, the conditional posterior distribution of $\beta$ given $\alpha$ in (5.22) cannot be reduced analytically to well known distributions and therefore it is impossible to sample directly by standard methods. So, to generate random numbers from this distribution, we use the Metropolis-Hastings method with normal proposal distribution, see Metropolis et al. [20]). Now, we suppose the next MCMC algorithm to draw samples from the posterior density (5.20) and in turn to obtain the Bayes estimates $\alpha$ and $\beta$ and any function of them such as $S(t)$ and $H(t)$ and the corresponding credible intervals.

Algorithm of MCMC method:

- $\quad$ Step 1. Take some initial guess of $\alpha$ and $\beta$, say $\alpha^{(0)}$ and $\beta^{(0)}$ respectively, $M=$ burn-in.

- $\quad$ Step 2. Set $j=1$.

- $\quad$ Step 3. Generate $\alpha^{(j)}$ from Gamma $\left(\alpha_{1}+R, b_{1}-\sum_{i=1}^{R} \ln \left(1-e^{-\beta^{j} x_{i}^{2}}\right)\right)$.

- $\quad$ Step 4. Using Metropolis-Hastings, generate $\beta^{(j)}$ from $\pi_{2}^{*}(\beta \mid \alpha, \underline{x})$ with the $N\left(\beta^{(j-1)}, \sigma^{2}\right)$ proposal distribution where $\sigma^{2}$ is the variance of $\beta$ obtained using variance-covariance matrix.

- $\quad$ Step 5. Compute $S(t)$ and $H(t)$ as

$$
\begin{gathered}
S(t)^{(j)}=1-\left(1-e^{-\beta^{(j)} t^{2}}\right)^{\alpha^{(j)}}, \\
H^{(j)}(t)=\frac{2 \alpha^{(j)} \beta^{(j)} t e^{-\beta^{(j)} t^{2}}\left(1-e^{-\beta^{(j)} t^{2}}\right)^{\alpha^{(j)}-1}}{1-\left(1-e^{-\beta(j)} t^{2}\right)^{\alpha^{(j)}}} .
\end{gathered}
$$

- $\quad$ Step 6. Set $j=j+1$.

- Step 7. Repeat steps 3-6 N times and obtain $\alpha^{(j)}, \beta^{(j)}, S^{(j)}(t)$ and $H^{(j)}(t), j=\mathrm{M}+1, \mathrm{~N}$.

- Step 8. To compute the credible intervals of $\alpha, \beta, S(t)$ and $H(t)$, order $\alpha^{(j)}, \beta^{(j)} ; S^{(j)}(t)$ and $H^{(j)}(t), j=M+1, \ldots, N$, as $\left(\alpha^{(1)}<\ldots, \alpha^{(N-M)}\right),\left(\beta^{(1)}<\ldots,<\beta^{(N-M)},\left(S^{(1)}(t)<\ldots, S^{(N-M)}(t)\right.\right.$ and $\left(H^{(1)}(t)<\ldots, H^{(N-M)}(t)\right)$. Then the $100(1-\gamma) \%$ credible interval of $\alpha, \beta, S(t)$ and $H(t)$ is $\left(\zeta_{(N-M)_{\gamma / 2}}, \zeta_{(N-M)_{(1-\gamma / 2)}}\right)$.

The Bayes estimates of $\zeta=[\alpha, \beta, S(t), H(t)]$, under squared error loss function are given by

$$
\hat{\zeta}_{B S}=E[\zeta \mid \underline{x}]=\frac{\sum_{i=M+1}^{N} \zeta^{(i)}}{N-M} .
$$

The Bayes estimates of $\zeta=[\alpha, \beta, S(t), H(t)]$, under LINEX loss function are given by

$$
\varepsilon_{B H}=-\frac{1}{a} \ln \left[\frac{\sum i-M+1 e^{-\alpha \zeta(x)}}{N-M}\right] \text {. }
$$

The Bayes estimates of $\zeta=[\alpha, \beta, S(t), H(t)]$, under general entropy loss function are given by

$$
\hat{S B C}=\left[\frac{\sum i=M+1\left[\zeta^{(i)}\right]^{-\alpha}}{N-M}\right]^{-\frac{1}{\alpha}} \text {. }
$$

\section{REAL LIFE DATA}

Wind power is renewable and environmentally friendly. It is an alternative clean energy source compared with the fossil fuels that pollute the lower layer of the atmosphere. Wind power is a form of solar energy, driven by the unequal heating of the earth's surface. The most important parameter of the wind power is wind speed. Statistical methods are useful for 
estimating wind speed because it is a random phenomenon. For this reason, wind speed probabilities can be modelled by using probability distributions. We have taken the daily average wind speeds from 1/1/2009 to 10 / 4 / 2009 for Cairo city as follows

$\begin{array}{cccccccccc}3.5 & 3.1 & 3.8 & 3.2 & 3.2 & 4.5 & 5.6 & 5.7 & 4.9 & 5.7 \\ 4.3 & 9.4 & 9.3 & 4.4 & 2.7 & 3.8 & 4.9 & 5.4 & 4.9 & 4.2 \\ 5.4 & 3.3 & 6.9 & 9.8 & 10 & 8 & 5.6 & 8.2 & 9.4 & 11.3 \\ 9.4 & 5.5 & 4.9 & 8.6 & 5 & 4.7 & 3.8 & 4.3 & 6.7 & 7.6 \\ 13.3 & 8.2 & 5.8 & 5.1 & 7.8 & 10.3 & 9.3 & 4.3 & 7.4 & 13.8 \\ 10.7 & 12 & 8.9 & 10.6 & 6.8 & 6.6 & 11.1 & 12.5 & 14.4 & 9.9 \\ 4.8 & 4.2 & 5.5 & 7.3 & 12.4 & 14.7 & 6.4 & 8.7 & 5.2 & 6.8 \\ 5.6 & 7.5 & 7.7 & 7.1 & 6.1 & 7.6 & 5.8 & 6.3 & 12.2 & 6 \\ 3.5 & 9.5 & 8.8 & 5.2 & 5 & 9.8 & 8 & 7.9 & 6.8 & 5.7 \\ 7.3 & 6.8 & 4.7 & 5.3 & 9.6 & 10.1 & 7.3 & 6.7 & 5.4 & 5.4\end{array}$

This data was produced by the national climatic data center (NCDC) in Asheville in the United States of America. Now, one of the most important subjects is type of distribution of any set of data will be known during statistical tests which are called the goodness of fit. We depended on Kolmogorov-Smirnov test to fit whether the data distribution as ER ( $\alpha, \beta)$ distribution or not. The calculated value of the K-S test is 0.0983022 for the ER $(\alpha, \beta)$ distribution and this value is smaller than their corresponding values expected at $5 \%$ significance level, which is 0.13403 at $n=100$. We have just plotted the empirical $S(t)$ and the fitted $S(t)$ in Figure1a. Observe that the ER $(\alpha, \beta)$ distribution can be a good model fitting this data. In Figure $1 b$ it shows that all the points of a $Q-Q$ plot are inside the unit square, so, it can be seen that the ER $(\alpha, \beta)$ distribution fits the data very well. P-value $=0.27052$, therefore, the high $\mathrm{p}$-value indicates that $\mathrm{ER}(\alpha, \beta)$ distribution can be used to analyze this data set. Now, we consider the case when the data are censored. We have six cases as following:

- $\quad$ Case I: $T_{1}=9.45, T_{2}=9.95, k=70, r=75$. In this case: $R=80, \mathrm{C}=T_{1}=9.45$.

- $\quad$ Case II: $T_{1}=9.45, T_{2}=9.95, k=70, r=83$. In this case: $R=83, C=x_{r: n}=9.8$.
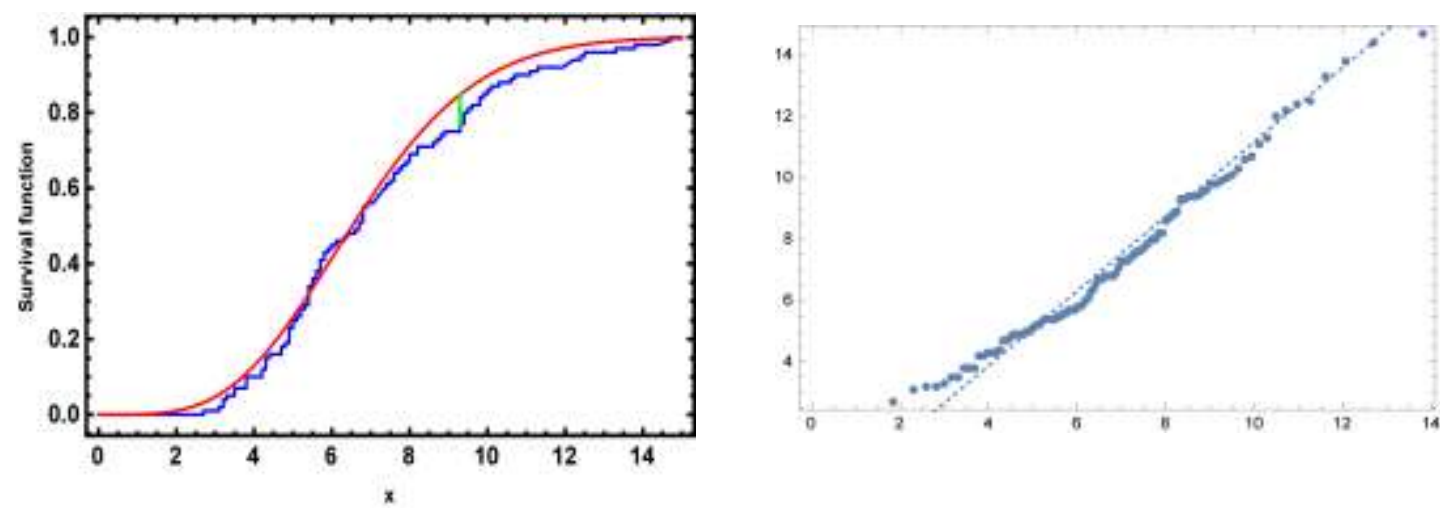

Figure 1: (a) Empirical and Fitted Survival Functions. (b) $Q$ - $Q$ plot compare data to a specific distribution.

- $\quad$ Case III: $T_{1}=9.45, T_{2}=9.95, k=70, r=86$. In this case: $R=85, C=\mathrm{T} 2=9.95$.

- Case IV: $T_{1}=8.4, T_{2}=10.5, k=80, r=87$. In this case: $R=87, C=X_{\mathrm{r}: \mathrm{n}}=10.1$.

- $\quad$ Case $V: T_{1}=8.4, T_{2}=10.5, k=84, r=90$. In this case: $R=88, C=T 2=10.5$.

- $\quad$ Case VI: $T_{1}=8.4, T_{2}=9.35, k=92, r=93$. In this case: $R=92, C=x_{k: n}=11.3$.

In all the six cases, we estimated the unknown parameters, we computed different estimates of the parameters $\alpha$ and $\beta$ in addition to $S(t)$ and $H(t)$. MLEs, Bootstrap confidence intervals (Boot-p, Boot-t) based on 1000 Bootstrap samples and Bayes estimates using MCMC method based on 11000 MCMC sample and discard the first 1000 values as 'burn-in'. To compute the MLEs, we used the numerical method and also computed the $95 \% \mathrm{ACl}$; the results are given in Table (1). Also, we computed the $95 \%$ ACl Bootstrap confidence intervals. The results are given in Table (2). To compute the Bayes estimates we considered the square error loss, LINEX loss and general entropy loss functions. Under non-informative priors the hyper parameters are $a_{1}=a_{2}=b_{1}=b_{2}=0$. The results are given in Table (3). Under the informative priors the hyper parameters are $a_{1}=0.9, a_{2}=0.8, b_{1}=0.5$ and $b_{2}=1.0$. The results are given in Table (4). Also, we computed the $95 \% \mathrm{ACl}$ based on the MCMC samples. The results are given in Table (5). The characteristics of the unknown parameters for MCMC method under non-informative and informative priors are given in Table (6) and Table (7) respectively. Figure 
(2) plot the MCMC output of $\alpha, \beta, S(t)$ and $H(t)$. The histogram of $\alpha, \beta, S(t)$ and $H(t)$ are given in Figure (3). In all the cases $\alpha=2$ and $\beta=0.026$ are considered.

Table 1: Estimation of $\alpha, \beta, S$ and $H$ and the $95 \%$ confidence interval of MLE method for six cases of UHCS

\begin{tabular}{|c|c|c|c|c|c|}
\hline Cases & Parameters & MLE & Lower & Upper & Length \\
\hline \multirow[t]{4}{*}{ Case I } & $\alpha$ & 2.2276 & 1.4839 & 2.9714 & 1.4875 \\
\hline & $\beta$ & 0.0281 & 0.0210 & 0.0352 & 0.0142 \\
\hline & $S$ & 0.9646 & 0.9373 & 0.9919 & 0.0546 \\
\hline & $H$ & 0.0479 & 0.0231 & 0.0727 & 0.0496 \\
\hline \multirow[t]{4}{*}{ Case II } & $\alpha$ & 2.2150 & 1.4873 & 2.9427 & 1.4554 \\
\hline & $\beta$ & 0.0279 & 0.0210 & 0.0348 & 0.0138 \\
\hline & $s$ & 0.9643 & 0.9370 & 0.9916 & 0.0546 \\
\hline & $H$ & 0.0481 & 0.0233 & 0.0728 & 0.0494 \\
\hline \multirow[t]{4}{*}{ Case III } & $\alpha$ & 2.2426 & 1.5121 & 2.9732 & 1.4611 \\
\hline & $\beta$ & 0.0282 & 0.0214 & 0.0351 & 0.0137 \\
\hline & $s$ & 0.9650 & 0.9381 & 0.9918 & 0.0537 \\
\hline & $H$ & 0.0477 & 0.0230 & 0.0724 & 0.0494 \\
\hline \multirow[t]{4}{*}{ Case IV } & $\alpha$ & 2.2724 & 1.5382 & 3.0067 & 1.4686 \\
\hline & $\beta$ & 0.0286 & 0.0218 & 0.0354 & 0.0136 \\
\hline & $s$ & 0.9656 & 0.9392 & 0.9920 & 0.0529 \\
\hline & $H$ & 0.0473 & 0.0227 & 0.0719 & 0.0493 \\
\hline \multirow[t]{4}{*}{ Case V } & $\alpha$ & 2.2084 & 1.5001 & 2.9167 & 1.4166 \\
\hline & $\beta$ & 0.0278 & 0.0212 & 0.0344 & 0.0132 \\
\hline & $s$ & 0.9642 & 0.9370 & 0.9913 & 0.0543 \\
\hline & $H$ & 0.0482 & 0.0235 & 0.0728 & 0.0493 \\
\hline \multirow[t]{4}{*}{ Case VI } & $\alpha$ & 2.1828 & 1.4959 & 2.8697 & 1.3739 \\
\hline & $\beta$ & 0.0275 & 0.0212 & 0.0339 & 0.0127 \\
\hline & $s$ & 0.9635 & 0.9363 & 0.9908 & 0.0545 \\
\hline & $H$ & 0.0485 & 0.0239 & 0.0731 & 0.0491 \\
\hline
\end{tabular}

Table 2: Estimation of $\alpha, \beta, S$ and $H$ and the $95 \%$ confidence interval of Boot-P and Boot-T methods for six cases of UHCS

\begin{tabular}{lcllllllll}
\hline Cases & Parameters & Boot-P & Lower & Upper & Length & Boot-P & Lower & Upper & Length \\
\hline Case I & $\alpha$ & 2.2648 & 1.8221 & 2.8330 & 1.0109 & 2.2461 & 1.8120 & 2.8045 & 0.9924 \\
& $\beta$ & 0.0282 & 0.0242 & 0.0326 & 0.0084 & 0.0277 & 0.0245 & 0.0307 & 0.0061 \\
& $S$ & 0.9644 & 0.9420 & 0.9830 & 0.0410 & 0.9641 & 0.9474 & 0.9897 & 0.0422 \\
& $H$ & 0.0106 & 0.0105 & 0.0113 & 0.0008 & 0.0485 & 0.0276 & 0.0647 & 0.0371 \\
Case II & $\alpha$ & 2.2457 & 1.8314 & 2.8450 & 1.0136 & 2.2744 & 1.8247 & 2.8740 & 1.0493 \\
& $\beta$ & 0.0280 & 0.0242 & 0.0326 & 0.0083 & 0.0278 & 0.0239 & 0.0311 & 0.0071 \\
& $S$ & 0.9638 & 0.9429 & 0.9829 & 0.0400 & 0.9646 & 0.9486 & 0.9903 & 0.0416 \\
& $H$ & 0.0106 & 0.0104 & 0.0160 & 0.0055 & 0.0479 & 0.0268 & 0.0640 & 0.0372 \\
Case III & $\alpha$ & 2.2844 & 1.8609 & 2.8806 & 1.0196 & 2.3096 & 1.7632 & 2.9003 & 1.1370
\end{tabular}


Volume 12 Number 12

Journal of Advances in Mathematics

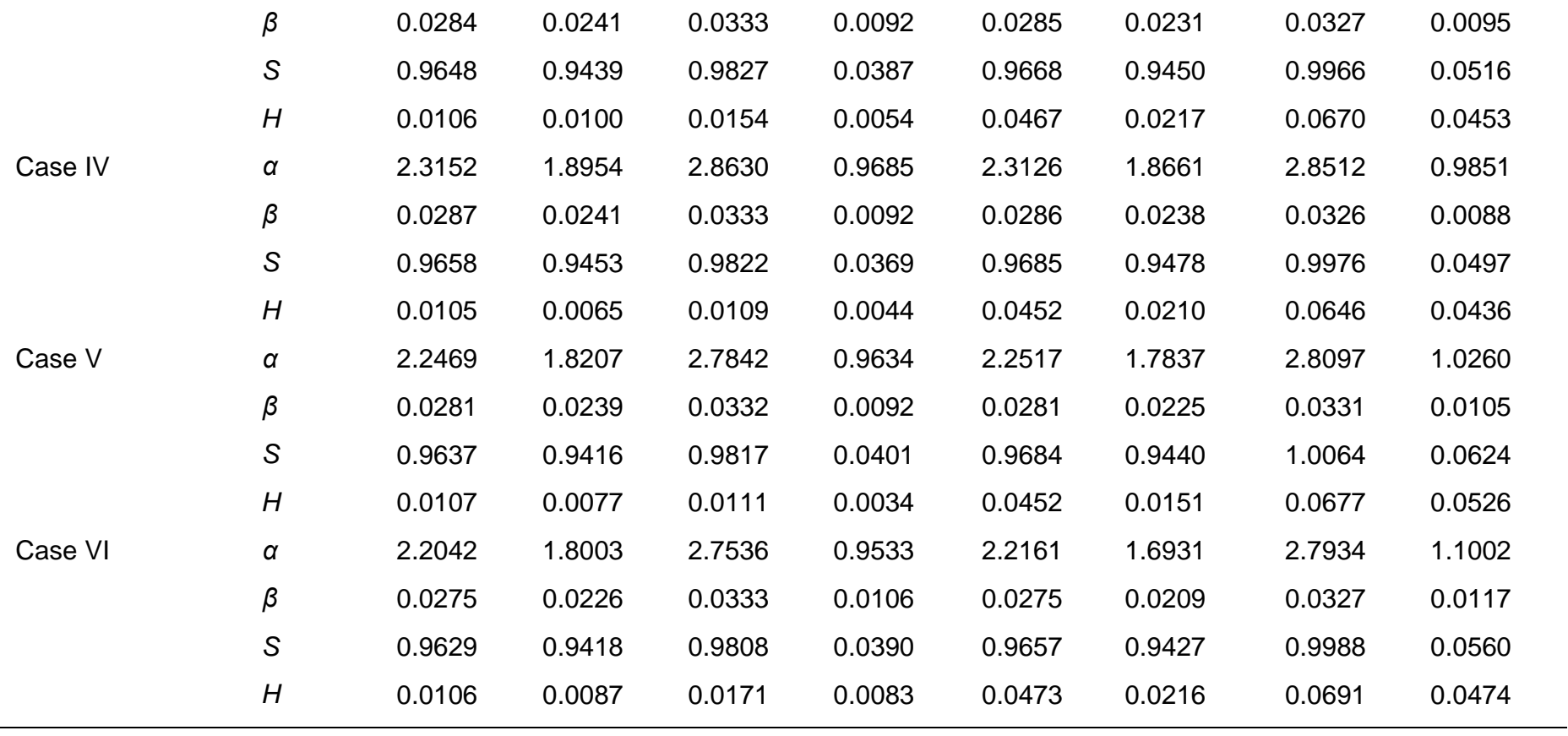


Table 3: Estimation of $\alpha, \beta, S$ and $\mathrm{H}$ for MCMC method for six cases of UHCS with non-informative prior

\begin{tabular}{|c|c|c|c|c|c|c|c|c|}
\hline \multirow{2}{*}{ Cases } & \multirow{2}{*}{ Parameters } & \multirow{2}{*}{ SE } & \multicolumn{3}{|c|}{ LINEX } & \multicolumn{3}{|c|}{ GE } \\
\hline & & & $a=-4$ & $a=0.5$ & $a=4$ & $a=-4$ & $a=0.5$ & $a=4$ \\
\hline \multirow[t]{4}{*}{ Case I } & $\alpha$ & 1.2877 & 1.4664 & 1.2688 & 1.1723 & 1.3757 & 1.2520 & 1.1667 \\
\hline & $\beta$ & 0.0194 & 0.0194 & 0.0194 & 0.0194 & 0.0205 & 0.0190 & 0.0178 \\
\hline & $S$ & 0.8999 & 0.9019 & 0.8996 & 0.8977 & 0.9017 & 0.8991 & 0.8970 \\
\hline & $H$ & 0.0837 & 0.0842 & 0.0836 & 0.0831 & 0.0885 & 0.0814 & 0.0752 \\
\hline \multirow[t]{4}{*}{ Case II } & $\alpha$ & 1.3806 & 1.5549 & 1.3612 & 1.2603 & 1.4634 & 1.3463 & 1.2626 \\
\hline & $\beta$ & 0.0204 & 0.0204 & 0.0204 & 0.0204 & 0.0212 & 0.0200 & 0.0191 \\
\hline & $s$ & 0.9100 & 0.9117 & 0.9098 & 0.9082 & 0.9115 & 0.9093 & 0.9076 \\
\hline & $H$ & 0.0794 & 0.0800 & 0.0794 & 0.0789 & 0.0845 & 0.0771 & 0.0705 \\
\hline \multirow[t]{4}{*}{ Case III } & $\alpha$ & 1.4912 & 1.7009 & 1.4684 & 1.3538 & 1.5822 & 1.4540 & 1.3652 \\
\hline & $\beta$ & 0.0217 & 0.0217 & 0.0217 & 0.0217 & 0.0226 & 0.0213 & 0.0203 \\
\hline & $s$ & 0.9193 & 0.9208 & 0.9191 & 0.9178 & 0.9207 & 0.9188 & 0.9173 \\
\hline & $H$ & 0.0755 & 0.0760 & 0.0754 & 0.0750 & 0.0809 & 0.0730 & 0.0660 \\
\hline \multirow[t]{4}{*}{ Case IV } & $\alpha$ & 1.6093 & 1.8518 & 1.5835 & 1.4523 & 1.7044 & 1.5700 & 1.4742 \\
\hline & $\beta$ & 0.0230 & 0.0231 & 0.0230 & 0.0230 & 0.0239 & 0.0227 & 0.0216 \\
\hline & $S$ & 0.9279 & 0.9292 & 0.9277 & 0.9266 & 0.9290 & 0.9274 & 0.9261 \\
\hline & $H$ & 0.0716 & 0.0721 & 0.0715 & 0.0711 & 0.0772 & 0.0690 & 0.0617 \\
\hline \multirow[t]{4}{*}{ Case V } & $\alpha$ & 1.6108 & 1.8425 & 1.5850 & 1.4519 & 1.7051 & 1.5714 & 1.4736 \\
\hline & $\beta$ & 0.0228 & 0.0228 & 0.0228 & 0.0228 & 0.0236 & 0.0224 & 0.0215 \\
\hline & $s$ & 0.9290 & 0.9303 & 0.9288 & 0.9276 & 0.9301 & 0.9285 & 0.9272 \\
\hline & $H$ & 0.0705 & 0.0710 & 0.0704 & 0.0700 & 0.0762 & 0.0679 & 0.0606 \\
\hline \multirow[t]{4}{*}{ Case VI } & $\alpha$ & 1.7671 & 2.0264 & 1.7386 & 1.5927 & 1.8619 & 1.7274 & 1.6295 \\
\hline & $\beta$ & 0.0241 & 0.0241 & 0.0241 & 0.0240 & 0.0248 & 0.0237 & 0.0228 \\
\hline & $S$ & 0.9404 & 0.9414 & 0.9403 & 0.9394 & 0.9413 & 0.9401 & 0.9391 \\
\hline & $H$ & 0.0638 & 0.0642 & 0.0637 & 0.0633 & 0.0693 & 0.0612 & 0.0540 \\
\hline
\end{tabular}

Table 4: Estimation of $\alpha, \beta, S$ and $H$ for MCMC method for six cases of UHCS with informative prior 


\begin{tabular}{|c|c|c|c|c|c|c|c|c|}
\hline \multirow{2}{*}{ Cases } & \multirow{2}{*}{ Parameters } & \multirow{2}{*}{ SE } & \multicolumn{3}{|c|}{ LINEX } & \multicolumn{3}{|c|}{ GE } \\
\hline & & & $a=-4$ & $a=0.5$ & $a=4$ & $a=-4$ & $a=0.5$ & $a=4$ \\
\hline \multirow[t]{4}{*}{ Case I } & $\alpha$ & 1.3031 & 1.4883 & 1.2841 & 1.1874 & 1.3905 & 1.2678 & 1.1829 \\
\hline & $\beta$ & 0.0196 & 0.0197 & 0.0196 & 0.0196 & 0.0206 & 0.0192 & 0.0180 \\
\hline & $S$ & 0.9013 & 0.9033 & 0.9011 & 0.8992 & 0.9031 & 0.9006 & 0.8985 \\
\hline & $H$ & 0.0832 & 0.0838 & 0.0831 & 0.0827 & 0.0882 & 0.0809 & 0.0744 \\
\hline \multirow{4}{*}{ Case II } & $\alpha$ & 1.4274 & 1.6057 & 1.4071 & 1.3012 & 1.5108 & 1.3926 & 1.3071 \\
\hline & $\beta$ & 0.0211 & 0.0211 & 0.0211 & 0.0211 & 0.0220 & 0.0207 & 0.0197 \\
\hline & $S$ & 0.9135 & 0.9151 & 0.9133 & 0.9118 & 0.9149 & 0.9129 & 0.9112 \\
\hline & $H$ & 0.0784 & 0.0789 & 0.0783 & 0.0779 & 0.0836 & 0.0761 & 0.0696 \\
\hline \multirow[t]{4}{*}{ Case III } & $\alpha$ & 1.5250 & 1.7386 & 1.5015 & 1.3785 & 1.6153 & 1.4871 & 1.3922 \\
\hline & $\beta$ & 0.0222 & 0.0222 & 0.2220 & 0.0221 & 0.0231 & 0.0218 & 0.0207 \\
\hline & $S$ & 0.9215 & 0.9230 & 0.9213 & 0.9200 & 0.9228 & 0.9210 & 0.9185 \\
\hline & $H$ & 0.0748 & 0.0753 & 0.0747 & 0.0743 & 0.0801 & 0.0724 & 0.0657 \\
\hline \multirow[t]{4}{*}{ Case IV } & $\alpha$ & 1.6407 & 1.8910 & 1.6142 & 1.4786 & 1.7362 & 1.6010 & 1.5031 \\
\hline & $\beta$ & 0.0235 & 0.0235 & 0.0235 & 0.0235 & 0.0244 & 0.0231 & 0.0221 \\
\hline & $S$ & 0.9296 & 0.9309 & 0.9295 & 0.9283 & 0.9307 & 0.9292 & 0.9279 \\
\hline & $H$ & 0.0710 & 0.0715 & 0.0709 & 0.0705 & 0.0765 & 0.0684 & 0.0612 \\
\hline \multirow[t]{4}{*}{ Case V } & $\alpha$ & 1.6292 & 1.8782 & 1.6044 & 1.4737 & 1.7181 & 1.5917 & 1.4972 \\
\hline & $\beta$ & 0.0231 & 0.0231 & 0.0231 & 0.0231 & 0.0239 & 0.0227 & 0.0218 \\
\hline & $S$ & 0.9302 & 0.9314 & 0.9301 & 0.9290 & 0.9313 & 0.9298 & 0.9286 \\
\hline & $H$ & 0.0701 & 0.0706 & 0.0700 & 0.0696 & 0.0756 & 0.0676 & 0.0609 \\
\hline \multirow[t]{4}{*}{ Case VI } & $\alpha$ & 1.8038 & 2.1435 & 1.7736 & 1.6244 & 1.9060 & 1.7629 & 1.6644 \\
\hline & $\beta$ & 0.0246 & 0.0246 & 0.0246 & 0.0246 & 0.0254 & 0.0243 & 0.0234 \\
\hline & $S$ & 0.9418 & 0.9427 & 0.9417 & 0.9409 & 0.9426 & 0.9415 & 0.9406 \\
\hline & $H$ & 0.0633 & 0.0638 & 0.0632 & 0.0628 & 0.0689 & 0.0606 & 0.0527 \\
\hline
\end{tabular}


Table 5: The $95 \%$ confidence interval of MCMC for $\alpha, \beta, S$ and $H$ for six cases of UHCS

\begin{tabular}{|c|c|c|c|c|c|c|c|}
\hline \multirow[t]{2}{*}{ Cases } & \multirow[t]{2}{*}{ Parameters } & \multicolumn{3}{|c|}{ MCMC non informative } & \multicolumn{3}{|c|}{ MCMC with informative } \\
\hline & & Lower & Upper & Length & Lower & Upper & Length \\
\hline \multirow[t]{4}{*}{ Case I } & $\alpha$ & 0.9372 & 1.7064 & 0.7692 & 0.9541 & 1.7245 & 0.7704 \\
\hline & $\beta$ & 0.0145 & 0.0250 & 0.0105 & 0.0146 & 0.0251 & 0.0105 \\
\hline & $S$ & 0.8481 & 0.9425 & 0.0944 & 0.8502 & 0.9434 & 0.0932 \\
\hline & $H$ & 0.0604 & 0.1083 & 0.0479 & 0.0598 & 0.1081 & 0.0483 \\
\hline \multirow[t]{4}{*}{ Case II } & $\alpha$ & 0.0196 & 1.8074 & 0.7878 & 1.0594 & 1.8641 & 0.8047 \\
\hline & $\beta$ & 0.0157 & 0.0254 & 0.0098 & 0.0163 & 0.0264 & 0.0101 \\
\hline & $s$ & 0.8621 & 0.9491 & 0.0871 & 0.8674 & 0.9512 & 0.0839 \\
\hline & $H$ & 0.0565 & 0.1044 & 0.0479 & 0.0552 & 0.1030 & 0.0477 \\
\hline \multirow[t]{4}{*}{ Case III } & $\alpha$ & 1.1035 & 1.9516 & 0.8481 & 1.1258 & 1.9803 & 0.8545 \\
\hline & $\beta$ & 0.0168 & 0.0270 & 0.0102 & 0.0170 & 0.0276 & 0.0107 \\
\hline & $s$ & 0.8748 & 0.9556 & 0.0808 & 0.8775 & 0.9562 & 0.0787 \\
\hline & $H$ & 0.0528 & 0.1003 & 0.0475 & 0.0523 & 0.0995 & 0.0472 \\
\hline \multirow[t]{4}{*}{ Case IV } & $\alpha$ & 1.1940 & 2.0899 & 0.8960 & 1.2232 & 2.1355 & 0.9123 \\
\hline & $\beta$ & 0.0179 & 0.0285 & 0.0107 & 0.0184 & 0.0290 & 0.0106 \\
\hline & $S$ & 0.8862 & 0.9608 & 0.0747 & 0.8886 & 0.9616 & 0.0730 \\
\hline & $H$ & 0.0489 & 0.0964 & 0.0474 & 0.0483 & 0.0959 & 0.0476 \\
\hline \multirow[t]{4}{*}{ Case V } & $\alpha$ & 1.1956 & 2.1037 & 0.9082 & 1.2190 & 2.0971 & 0.8781 \\
\hline & $\beta$ & 0.0180 & 0.0280 & 0.0101 & 0.0182 & 0.0283 & 0.0101 \\
\hline & $S$ & 0.8869 & 0.9623 & 0.0754 & 0.8895 & 0.9615 & 0.0720 \\
\hline & $H$ & 0.0477 & 0.0954 & 0.0477 & 0.0483 & 0.0950 & 0.0466 \\
\hline \multirow[t]{4}{*}{ Case VI } & $\alpha$ & 1.3258 & 2.2754 & 0.9496 & 1.3662 & 2.3289 & 0.9627 \\
\hline & $\beta$ & 0.0192 & 0.0292 & 0.0101 & 0.0196 & 0.0297 & 0.0101 \\
\hline & $s$ & 0.9042 & 0.9687 & 0.0644 & 0.9066 & 0.9698 & 0.0632 \\
\hline & $H$ & 0.0423 & 0.0876 & 0.0453 & 0.0415 & 0.0870 & 0.0455 \\
\hline
\end{tabular}

Table 6: The characteristics for $\alpha, \beta, S$ and $H$ for six cases of UHCS with non- informative prior 


\begin{tabular}{|c|c|c|c|c|c|c|c|}
\hline Cases & Parameters & Mean & Median & Mode & Standard deviation & Root MS & Skewness \\
\hline \multirow[t]{4}{*}{ Case I } & $\alpha$ & 1.2878 & 1.2659 & 1.2530 & 0.2362 & 1.3092 & 0.5488 \\
\hline & $\beta$ & 0.0195 & 0.0193 & 0.0190 & 0.0032 & 0.0197 & 0.2662 \\
\hline & $S$ & 0.8999 & 0.9023 & 0.9153 & 0.0288 & 0.9004 & -0.5123 \\
\hline & $H$ & 0.0837 & 0.0834 & 0.0846 & 0.0146 & 0.0850 & 0.1477 \\
\hline \multirow[t]{4}{*}{ Case II } & $\alpha$ & 1.3807 & 1.3627 & 1.3620 & 0.2386 & 1.4011 & 0.4624 \\
\hline & $\beta$ & 0.0204 & 0.0203 & 0.0203 & 0.0030 & 0.0207 & 0.1913 \\
\hline & $S$ & 0.9101 & 0.9126 & 0.9259 & 0.0266 & 0.9104 & -0.5727 \\
\hline & $H$ & 0.0795 & 0.0792 & 0.0824 & 0.0145 & 0.0808 & 0.1718 \\
\hline \multirow[t]{4}{*}{ Case III } & $\alpha$ & 1.4913 & 1.4666 & 1.3370 & 0.2591 & 1.5136 & 0.5113 \\
\hline & $\beta$ & 0.0219 & 0.0216 & 0.0209 & 0.0032 & 0.0220 & 0.2348 \\
\hline & $S$ & 0.9194 & 0.9218 & 0.9235 & 0.0248 & 0.9197 & -0.5614 \\
\hline & $H$ & 0.0756 & 0.0750 & 0.0740 & 0.0146 & 0.0770 & 0.1914 \\
\hline \multirow[t]{4}{*}{ Case IV } & $\alpha$ & 1.6094 & 1.5891 & 1.5350 & 0.2759 & 1.6329 & 0.4636 \\
\hline & $\beta$ & 0.0231 & 0.0230 & 0.0230 & 0.0032 & 0.0233 & 0.1440 \\
\hline & $S$ & 0.9280 & 0.9302 & 0.9321 & 0.0230 & 0.9282 & -0.6200 \\
\hline & $H$ & 0.0717 & 0.0714 & 0.0740 & 0.0145 & 0.0731 & 0.2225 \\
\hline \multirow[t]{4}{*}{ Case V } & $\alpha$ & 1.6108 & 1.5894 & 1.5400 & 0.2756 & 1.6343 & 0.4386 \\
\hline & $\beta$ & 0.0228 & 0.0228 & 0.0228 & 0.0031 & 0.0231 & 0.1288 \\
\hline & $S$ & 0.9291 & 0.9315 & 0.9377 & 0.0231 & 0.9293 & -0.6574 \\
\hline & $H$ & 0.0706 & 0.0699 & 0.0677 & 0.0146 & 0.0720 & 0.2386 \\
\hline \multirow[t]{4}{*}{ Case VI } & $\alpha$ & 1.7671 & 1.7467 & 1.6970 & 0.2894 & 1.7907 & 0.4213 \\
\hline & $\beta$ & 0.0241 & 0.0241 & 0.0229 & 0.0031 & 0.0243 & 0.1025 \\
\hline & $S$ & 0.9405 & 0.9429 & 0.9455 & 0.0198 & 0.9407 & -0.6924 \\
\hline & $H$ & 0.0638 & 0.0633 & 0.0674 & 0.0137 & 0.0653 & 0.2706 \\
\hline
\end{tabular}

Table 7: The characteristics for $\alpha, \beta, S$ and $H$ for six cases of UHCS with informative prior 
Journal of Advances in Mathematics

\begin{tabular}{|c|c|c|c|c|c|c|c|}
\hline Cases & Parameters & Mean & Median & Mode & Standard deviation & Root MS & Skewness \\
\hline \multirow{4}{*}{ Case I } & $\alpha$ & 1.3031 & 1.2827 & 1.2370 & 0.2363 & 1.3244 & 0.5675 \\
\hline & $\beta$ & 0.0197 & 0.0195 & 0.0189 & 0.0032 & 0.0199 & 0.3000 \\
\hline & $S$ & 0.9014 & 0.9040 & 0.9102 & 0.0286 & 0.9018 & -0.5398 \\
\hline & $H$ & 0.0833 & 0.0829 & 0.0850 & 0.0148 & 0.0846 & 0.1762 \\
\hline \multirow[t]{4}{*}{ Case II } & $\alpha$ & 1.4275 & 1.4092 & 1.3520 & 0.2439 & 1.4482 & 0.4364 \\
\hline & $\beta$ & 0.0211 & 0.0210 & 0.0215 & 0.0031 & 0.0214 & 0.1878 \\
\hline & $S$ & 0.9135 & 0.9165 & 0.9249 & 0.0258 & 0.9139 & -0.6006 \\
\hline & $H$ & 0.0785 & 0.0778 & 0.0766 & 0.0145 & 0.0798 & 0.2206 \\
\hline \multirow[t]{4}{*}{ Case III } & $\alpha$ & 1.5250 & 1.5079 & 104420 & 0.2625 & 1.5475 & 0.4247 \\
\hline & $\beta$ & 0.0222 & 0.0221 & 0.0213 & 0.0032 & 0.0224 & 0.1423 \\
\hline & $S$ & 0.9216 & 0.9243 & 0.9338 & 0.0243 & 0.9219 & -0.6532 \\
\hline & H & 0.0748 & 0.0744 & 0.0766 & 0.0144 & 0.0762 & 0.2155 \\
\hline \multirow[t]{4}{*}{ Case IV } & $\alpha$ & 1.6407 & 1.6178 & 1.5490 & 0.2792 & 1.6643 & 0.4624 \\
\hline & $\beta$ & 0.0235 & 0.0234 & 0.0229 & 0.0032 & 0.0238 & 0.2186 \\
\hline & $S$ & 0.9297 & 0.932 & 0.9366 & 0.0224 & 0.9300 & -0.644 \\
\hline & $H$ & 0.0710 & 0.0706 & 0.0704 & 0.0144 & 0.0725 & 0.2198 \\
\hline \multirow[t]{4}{*}{ Case V } & $\alpha$ & 1.6292 & 1.6163 & 1.5890 & 0.2691 & 1.6513 & 0.4022 \\
\hline & $\beta$ & 0.0231 & 0.0230 & 0.0230 & 0.0031 & 0.0233 & 0.1858 \\
\hline & $S$ & 0.9303 & 0.9333 & 0.9349 & 0.0223 & 0.9305 & -0.7608 \\
\hline & $H$ & 0.0701 & 0.0694 & 0.0648 & 0.0142 & 0.0716 & 0.3252 \\
\hline \multirow[t]{4}{*}{ Case VI } & $\alpha$ & 1.8038 & 1.7756 & 1.6670 & 0.2993 & 1.8285 & 0.5854 \\
\hline & $\beta$ & 0.0246 & 0.0245 & 0.0247 & 0.0031 & 0.0248 & 0.2061 \\
\hline & $S$ & 0.9419 & 0.9439 & 0.9447 & 0.0194 & 0.9421 & -0.6644 \\
\hline & $H$ & 0.0633 & 0.0629 & 0.0617 & 0.0137 & 0.0648 & 0.2263 \\
\hline
\end{tabular}
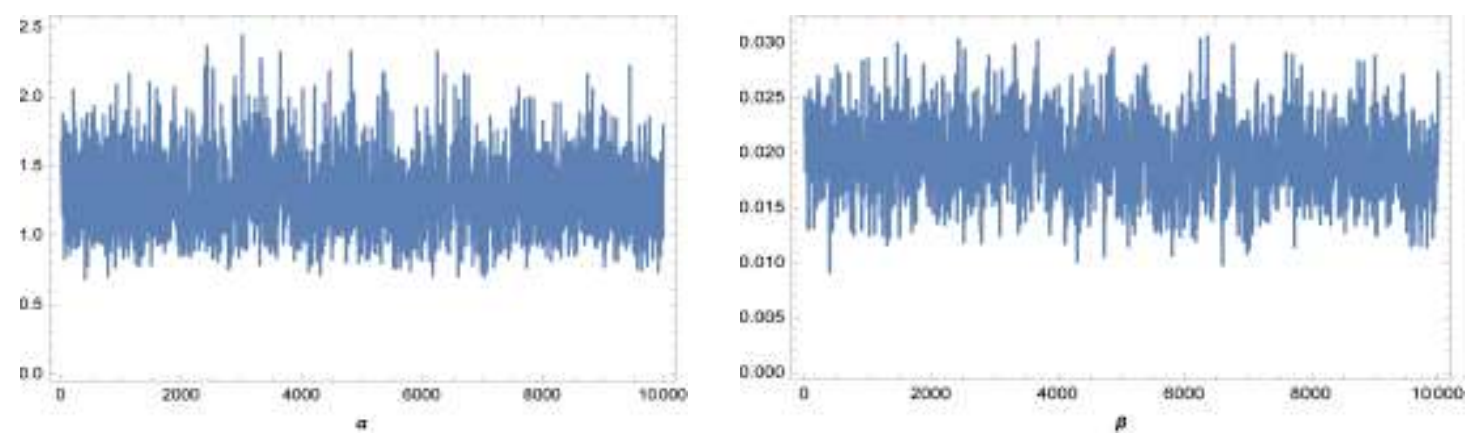

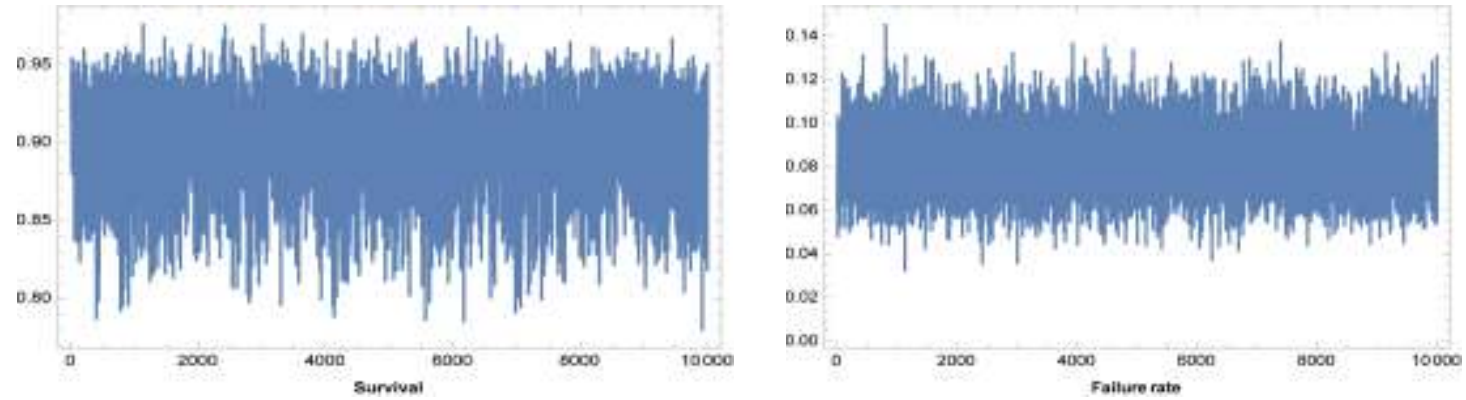

Figure 2: Trace plot of $\alpha, \beta, S(t)$ and $H(t)$ generated by MCMC method for case I.
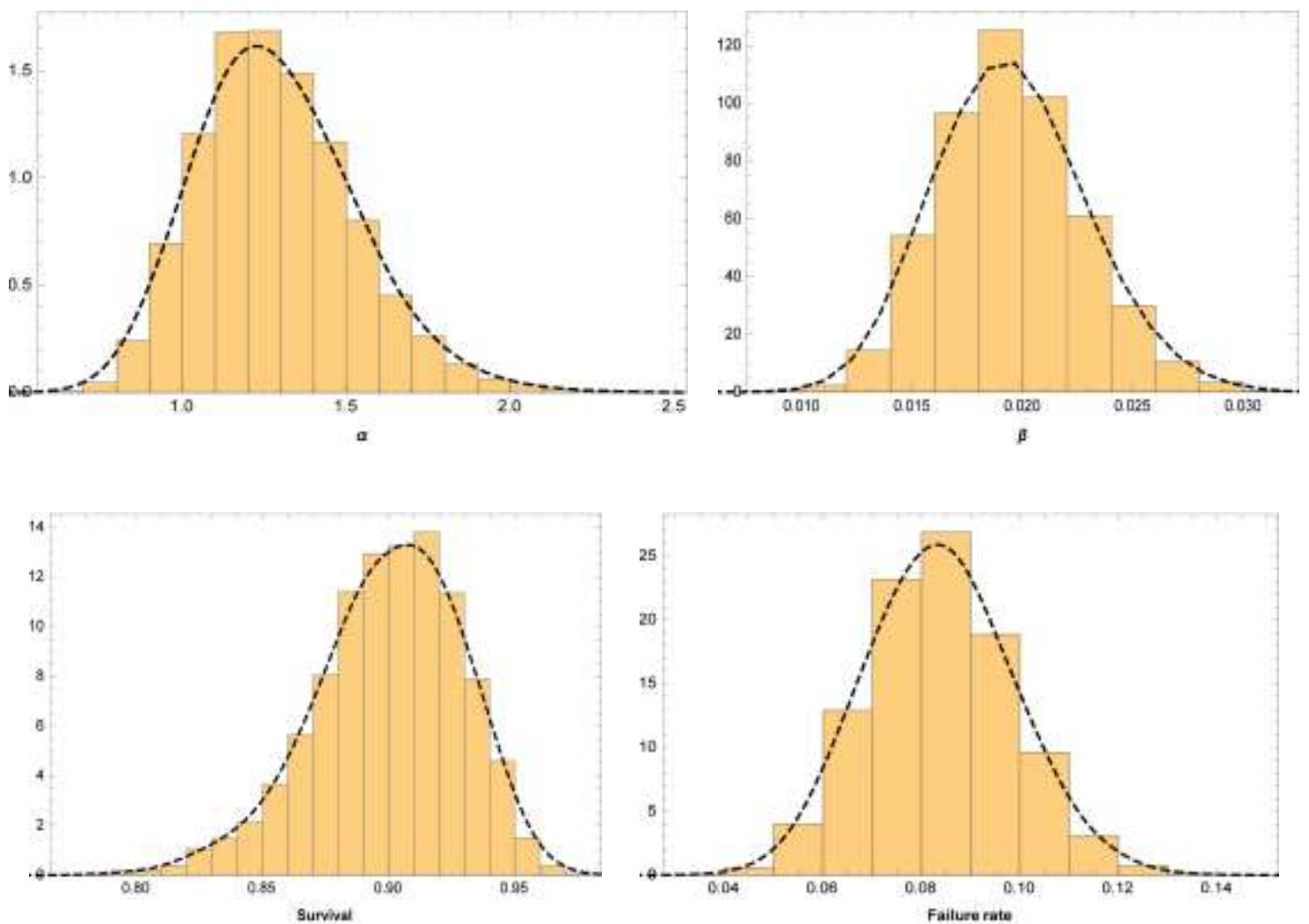

Figure 3: Histogram of $\alpha, \beta, S(t)$ and $H(t)$ generated by MCMC method for case I.

\section{CONCLUSION}

In this paper, we considered the Bayes estimation of the unknown parameters of the exponentiated Rayleigh distribution when the data is collected under the unified hybrid censored data. The MLEs, the bootstrap confidence intervals and the credible intervals based on the observed Fisher information matrix have been discussed. We supposed the gamma priors for both the unknown parameters and provided the Bayes estimators under the assumptions of squared error loss, LINEX loss and general entropy loss functions. In our study, the Bayes estimates cannot be obtained in explicit form. So, we used the MCMC technique to compute the approximate Bayes estimates and the corresponding credible intervals. We have applied the developed techniques on a real data set.

\section{REFERENCES}

[1] Abd-Elfattah, A. M. Goodness of _t test for the generalized Rayleigh distribution with unknown parameters, Journal of Statistical Computation and Simulation 81 (2011) 357-366.

[2] Ahmad, K. E., Fakhry, M. E., and Jaheen, Z. F. Empirical Bayes estimation of $P(Y<X)$ and characterization of Burrtype X model, Journal of Statistical Planning and Inference 64 (1997) 297-308. 
[3] Al-Kanani, I. H. and Jasim, S. A. Non-Bayesian and Bayesian estimation for generalized Rayleigh distribution, International Journal of Modern Mathematical Sciences 10 (2014) 103-115.

[4] Balakrishnan, N. and. Cohen, A. C. Order Statistics and Inference: Estimation Methods. Academic Press, San Diego, CA (1991).

[5] Balakrishnan, N. A synthesis of exact inferential results for exponential step-stress models and associated optimal accelerated life-tests, Metrika 69 (2009) 351-396.

[6] Burr, I. W. Cumulative frequency distribution, Annals of Mathematical Statistics 13 (1942) 215-232.

[7] Chandrasekar, B., Childs, A., and Balakrishnan, N. Exact likelihood inference for the exponential distribution under generalized Type-I and Type-II hybrid censoring, Naval Research Logistics 51 (2004) 994-1004.

[8] Childs, A. Chandrasekar, B., Balakrishnan, N., and Kundu, D. Exact likelihood inference based on Type-I and Type-II hybrid censored samples from the exponential distribution, Annals of the Institute of Statistical Mathematics 55 (2003) 319-330.

[9] Efron, B. The Jackknife, the Bootstrap and Other Resampling Plans, volume 38. CBMS/NSF Regional Conference Series in Applied Mathematics, SIAM, Philadelphia, PA, 1982.

[10] Epstein, B. Truncated life-test in exponential case, Annals of Mathematical Statistics 25 (1954) 555-564.

[11] Fathipour, P., Abolhasani, A., and Khamnei, H. J. Estimating $R=P(Y<X)$ in the generalized Rayleigh distribution with different scale parameters, Applied Mathematical Sciences 7 (2013) 87-92.

[12] Gupta, R. D., and Kundu, D. Hybrid censoring schemes with exponential failure distribution, Communications in Statistics Theory and Methods 27 (1998) 3065-3083.

[13] Hall, P. Theoretical comparison of bootstrap confidence intervals, Annals of Mathematical Statistics 16 (1988) 927953.

[14] Jaheen, Z. F. Empirical Bayes estimation of the reliability and failure rate functions of the Burr type $X$ failure model, Journal of Applied Statistics Science 3 (1996) 281-288.

[15] Jaheen, Z. F. Bayesian approach to prediction with outliers from the Burr type X model, Microelectronics Reliability 35 (1995) 45-47.

[16] Jeong, H. S., Park, J. I., and Yum, B. J. Development of $(r, T)$ hybrid sampling plans for exponential lifetime distributions, Journal of Applied Statistics 23 (1996) 601-607.

[17] Kundu, D. and. Raqab, M. Z. Generalized Rayleigh distribution: di_erent methods of estimation, Computational Statistics \& Data Analysis 49 (2005) 187-200.

[18] Lio, Y. L., Chen, D. G., and Tsai, T. R. Parameter estimations for generalized Rayleigh distribution under progressively Type-I interval censored data, American Open Journal of Statistics 1 (2011) 46-57.

[19] Mahmoud, M. A. W. and Ghazal, M. G. M. Estimations from the exponentiated rayleigh distribution based on generalized Type-II hybrid censored data, Journal of the Egyptian Mathematical Society (2016) 1-8.

[20] Metropolis, N., Rosenbluth, A. W., Rosenbluth, M. N., Teller, A. H., and Teller, E. Equations of state calculations by fast computing machines, Journal of Chemical and Physics 21 (1993) 1087-1091.

[21] MIL-STD-781-C. Reliability design quali_cation and production acceptance tests, exponential distribution. U.S. Government Printing Office, Washington, D. C., (1977).

[22] Mudholkar, G. S., Srivastava, D. K., and Freimer, M. The exponentiated Weibull family; a re-analysis of the bus motor failure data, Technometrics 37 (1995) 436-445.

[23] Nelson, W. Applied Life Data Analysis, John Wiley \& Sons, New York, NY.1982.

[24] Park, S. and Balakrishnan, N. On simple calculation of the Fisher information in hybrid censoring schemes, Statistics and Probability Letters 79 (2009) 1311-1319.

[25] Raqab, M. Z. Order statistics from the Burr type X model, Computers \& Mathematics with Applications 36 (1998) 111120.

[26] Raqab, M. Z. and Madi, M. T. Bayesian analysis for the exponentiated Rayleigh distribution, International Journal of Statistics LXVII (2009) 269-288.

[27] Raqab, M. Z. and Madi, M. T. Inference for the generalized Rayleigh distribution basedon progressively censored data, Journal of Statistical Planning and Inference 141 (2011) 3313-3322.

[28] Rayleigh, J. W. S. On the resultant of a large number of vibrations of the same pitch and of arbitrary phase, Philosophical Magazine and Journal of Science 10 (1880) 73-78.

[29] Sartawi, H. A. and Abu-Salih, M. S. Bayes prediction bounds for the Burr type X model, Communications in Statistics Theory and Methods 20 (1991) 2307-2330. 
[30] Shrestha, S. K. and Kumar, V. Bayesian analysis for the generalized Rayleigh distribution, International Journal of Statistika and Mathematika 9 (2014) 118-131.

[31] Surles, J. G. and Padgett, W. J. Inference for $P(Y<X)$ in the Burr Type $X$ model, Journal of Applied Statistics Science. (1998) 225-238.

[32] Surles, J. G. and Padgett, W. J. Inference for reliability and stress-strength for a scaled Burr Type X distribution, Lifetime Data Analysis. 7 (2001) 187-200.

[33] Upadhyay, S. K., Vasishta, N., and Smith, A. F. M. Bayes inference in life testing and reliability via Markov chain Monte Carlo simulation, Sankhya A, 63 (2001) 15-40.

[34]. Upadhyay, S. K. and Gupta, A Bayes analysis of modi_ed Weibull distribution via Markov chain Monte Carlo simulation, Journal of Statistical Computation and Simulation 80 (2010) 241-254.

[35] Vodã, V. G. Inferential procedures on a generalized Rayleigh variate, Applications of Mathe-matics 21 (1976) 395412. 